\title{
Combined QCD and Electro-Weak Fits at HERA
}
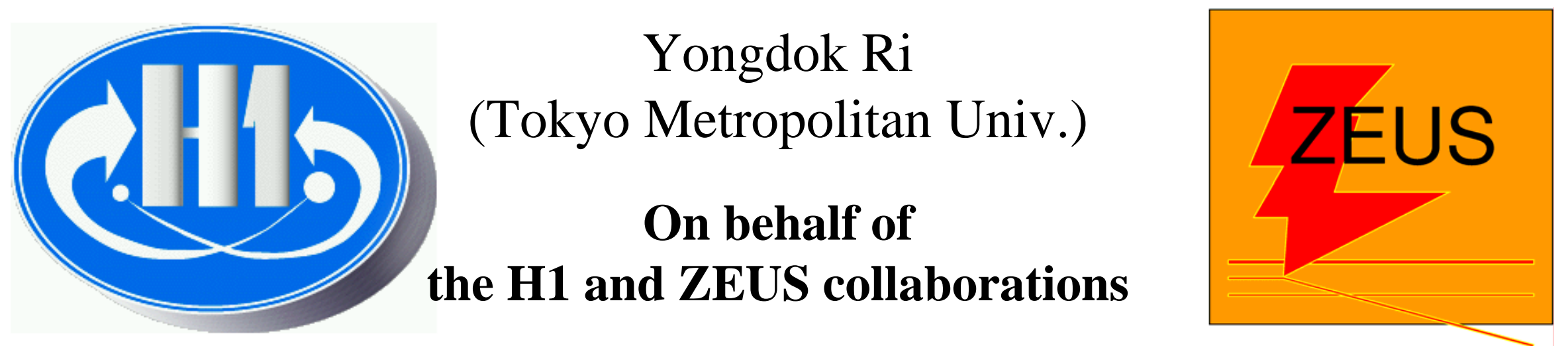

XXXIII International Conference on High Energy Physics

July 26 - August 2, 2006, Moscow, Russia

\section{Contents}

Deep Inelastic Scattering at HERA

Combined QCD and EW Fits

Results : $M_{W}$, quark couplings to $Z$ 


\section{HERA is first and unique ep collider in the world!}

$$
E_{p}=920 G e V, E_{e^{ \pm}}=27.5 \mathrm{GeV}, \sqrt{\mathrm{s}}=318 \mathrm{GeV}
$$

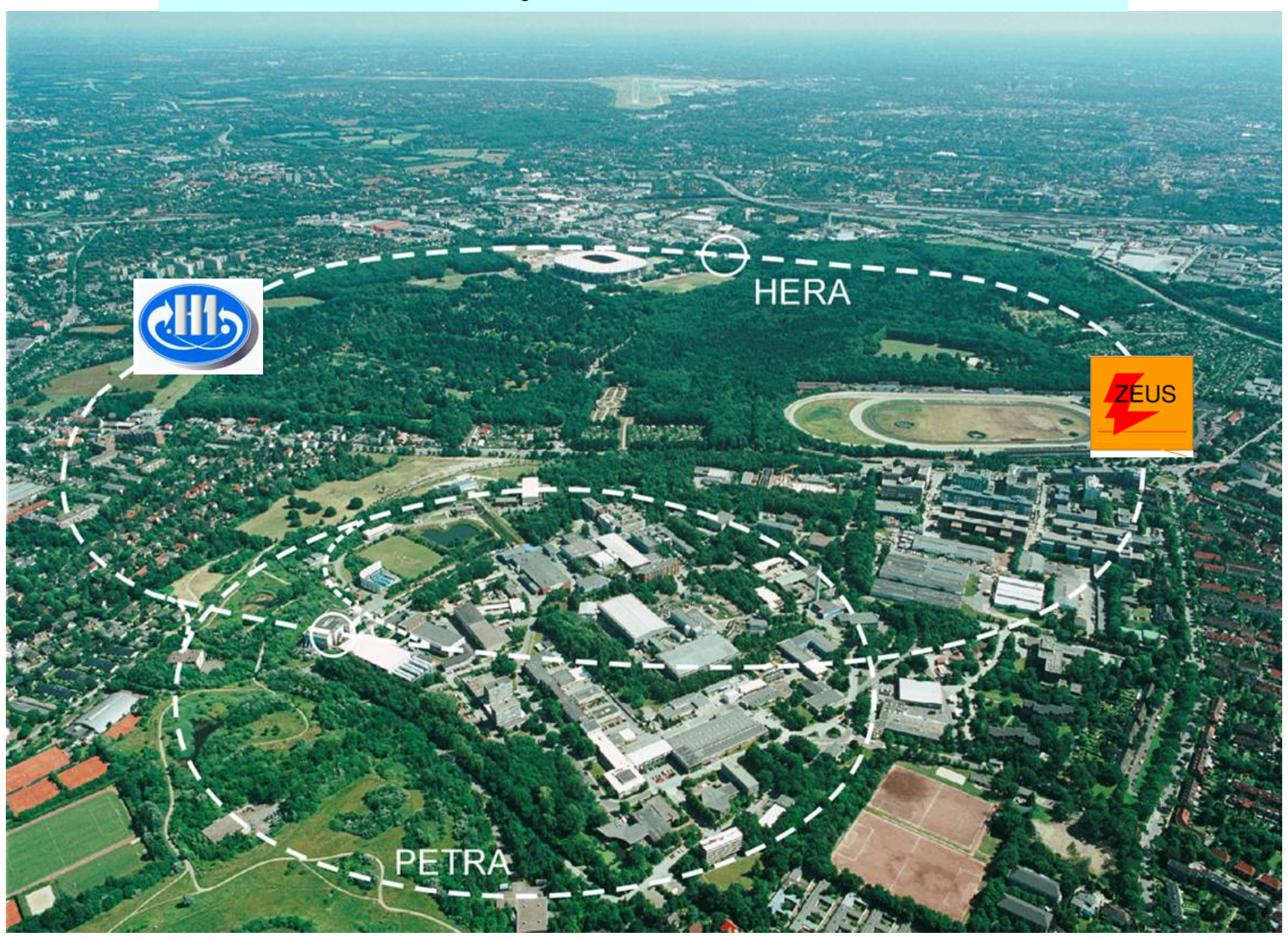

Two collider experiments, H1 and ZEUS, will run until 30/Jul/2007 


\section{Deep Inelastic Scattering at HERA}

Owing to the large center-of-mass energy, the electromagnetic and weak interactions become of comparable strength at HERA

Therefore both Neutral Current (NC), mediated by $\gamma$ or $\mathrm{Z}^{0}$, and Charged Current (CC), mediated by $\mathrm{W}^{ \pm}$, can occur

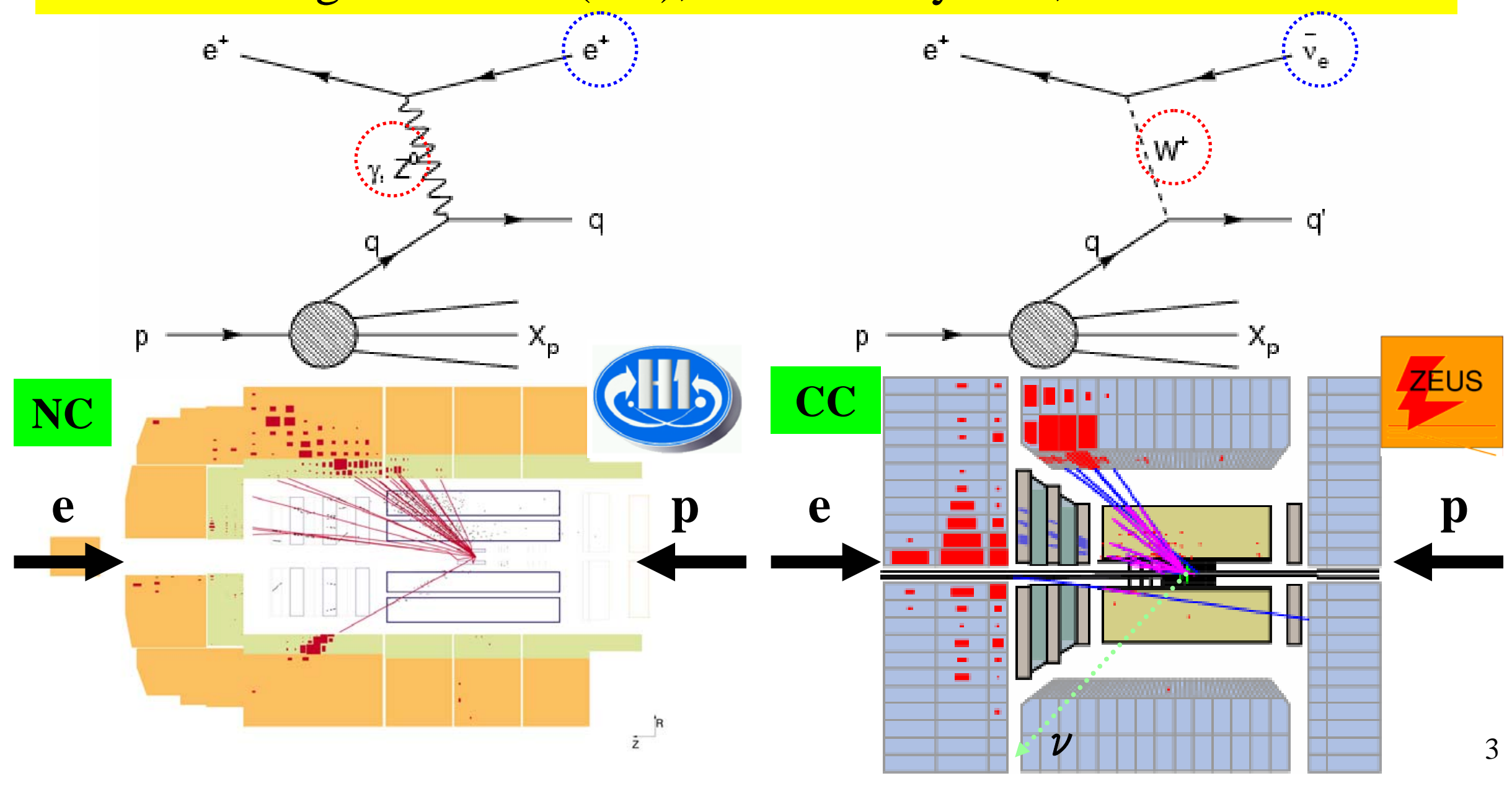




\section{DIS cross sections}

$\mathbf{P}(\mathbf{p})$
$\longrightarrow$

$$
\sigma_{\text {ep }}=\text { Coupling } \times \text { Propagator } \times \text { Kinematic Factor } \times \text { SFs }
$$

SFs $=$ coupling to boson $\times$ Parton Distribution Functions $($ PDFs)

DIS is a convoluted phenomenon for EW and QCD
Fit to the measured cross sections to study QCD and EW 


\section{Combined QCD and EW Fits}

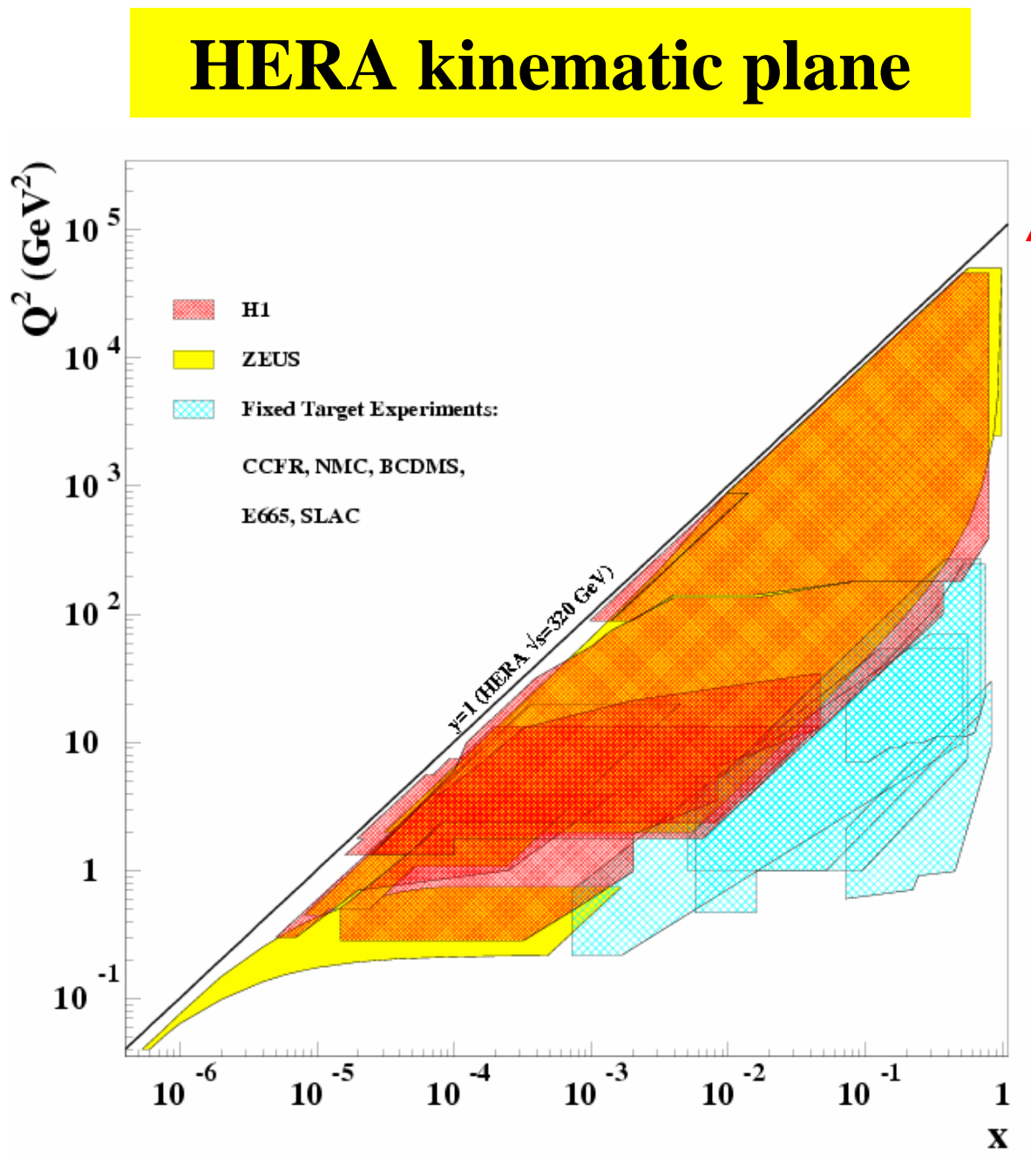

(3) Based on own knowledge of PDFs, EW parameters can be extracted at high- $Q^{2}$

(2) Perturbative QCD can predict the $\mathbf{Q}^{2}$ evolution of PDFs, DGLAP equation

Such a unique study, the simultaneous determination on PDFs and EW parameters, is only possible at HERA! 


\section{Extraction of PDFs for both experiments}

$>$ Both experiments fit only to their own data

-to handle the systematic errors within single experiment

-to eliminate the uncertainty from heavy-target correction

$>$ DGLAP evolution equations were performed in $\overline{\mathbf{M S}}$ renormalization scheme

\begin{tabular}{|c|c|c|}
\hline & H1 PDF 2000 & ZEUS-JETs ZÉus \\
\hline Data-sets & $\begin{aligned} \text { HERA-I } & \mathrm{F}_{2} \\
& + \text { Unpol. highQ }{ }^{2} \mathrm{NC}+\mathrm{CC}\end{aligned}$ & 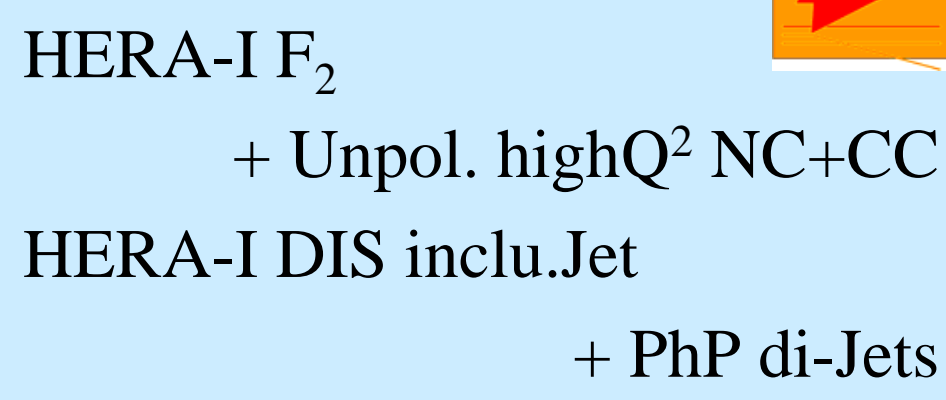 \\
\hline $\begin{array}{c}\text { PDFs } \\
\text { parameterization }\end{array}$ & $\begin{array}{l}x g, x U, x D, x \bar{U}, x \bar{D} \\
\text { are parameterised at } \mathrm{Q}_{0}{ }^{2}=4 \mathrm{GeV}^{2}\end{array}$ & $\begin{array}{l}u_{v}, d_{v}, \text { Sea } \text {, gluon, } x \Delta(=x \bar{d}-x \bar{u}) \\
\text { are parameterised at } \mathrm{Q}_{0}{ }^{2}=7 \mathrm{GeV}^{2}\end{array}$ \\
\hline $\begin{array}{l}\text { Treatment of } \\
\text { correlated } \\
\text { systematic } \\
\text { uncertainties }\end{array}$ & $\begin{array}{l}\text { evaluated using } \\
\qquad \text { Hessian method }\end{array}$ & $\begin{array}{l}\text { evaluated using } \\
\qquad \text { OFFSET method }\end{array}$ \\
\hline
\end{tabular}




\section{HERA-II}

Since Autumn 2003, HERA-II started with two upgrade :

$>$ Large luminosity $\rightarrow$ more sensitivity at high-Q ${ }^{2}$

$>$ Polarized e beams $\rightarrow$ direct sensitivity on EW

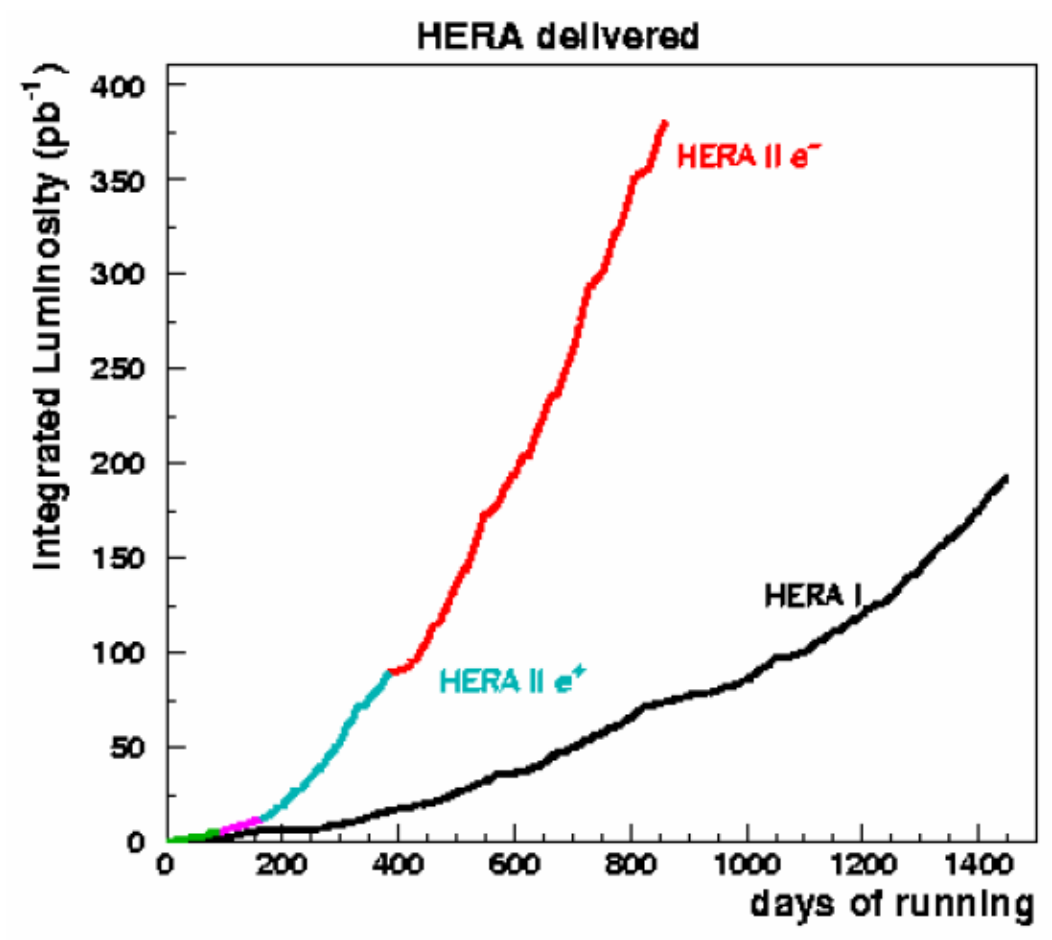

\begin{tabular}{|c|c|c|}
\hline & HERA-I & HERA-II \\
\hline $\mathrm{e}^{+}$ & $\sim 100 \mathrm{pb}^{-1}$ & $\sim 20 \mathrm{pb}^{-1} @ \mathrm{P}_{\mathrm{e}}=+33 \%$ \\
& & $\sim 20 \mathrm{pb}^{-1} @ \mathrm{P}_{\mathrm{e}}=-40 \%$ \\
\hline $\mathrm{e}^{-}$ & $\sim 20 \mathrm{pb}^{-1}$ & $\sim 40 \mathrm{pb}^{-1} @ \mathrm{P}_{\mathrm{e}}=+35 \%$ \\
& & $\sim 80 \mathrm{pb}^{-1} @ \mathrm{P}_{\mathrm{e}}=-27 \%$ \\
\hline
\end{tabular}

Recently, H1 and ZEUS collaborations measured polarized ep CC/NC DIS cross sections in HERA-II! $\leftarrow$ See talks from Alex \& Vladimir 


\section{Fit including HERA-II data}

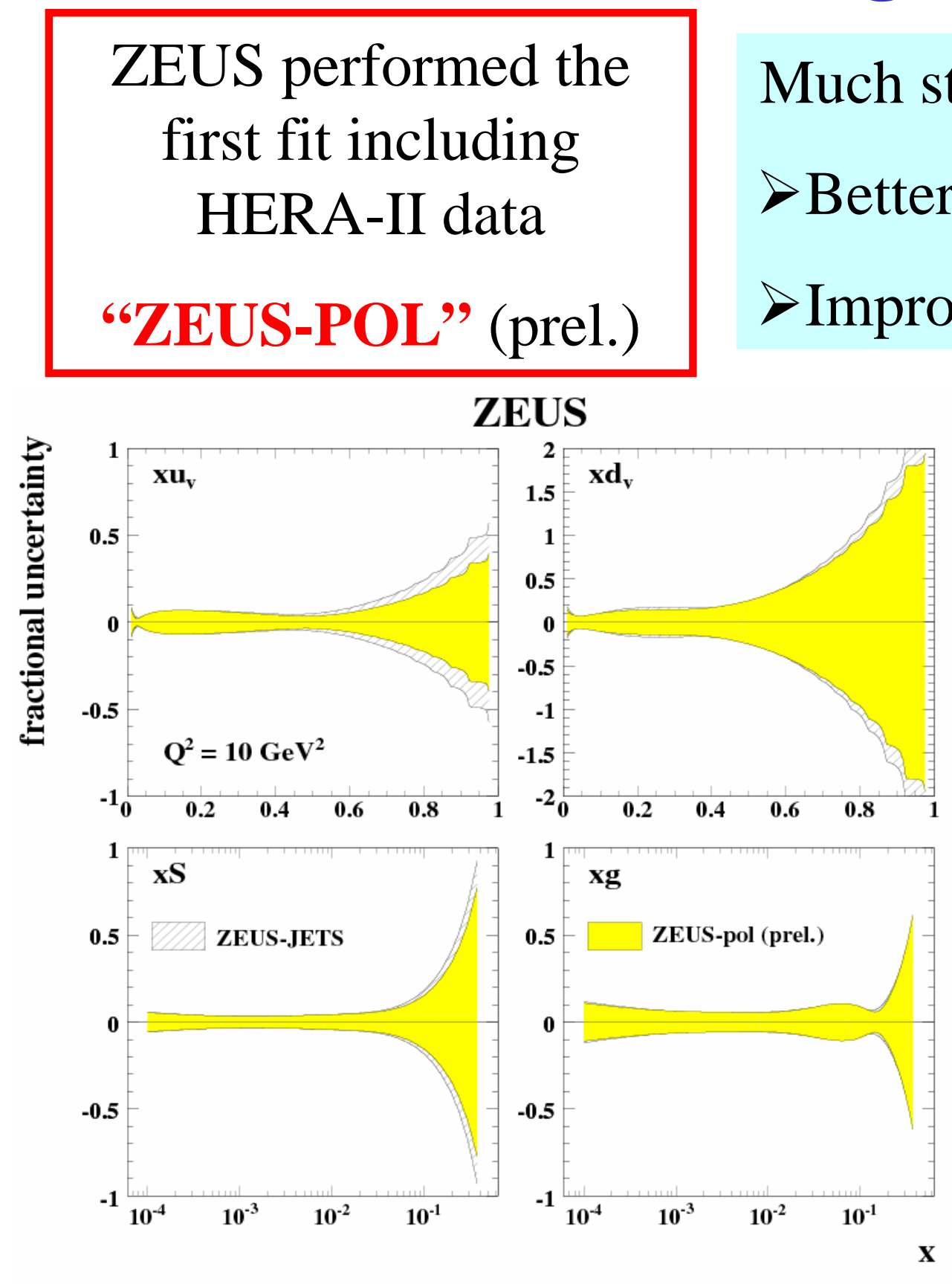

\section{Impact on PDFs :}

The precisions of the high-x PDFs are improved, particularly for the $\mathrm{u}$-valence PDF, and it can be expected from :

$$
\begin{aligned}
& \sigma_{N C} \propto 4 u+d \\
& \sigma_{C C} \propto u
\end{aligned}
$$

How is on EW parameters?

See next slides. 


\section{Extraction of $\mathbf{M}_{\mathbf{W}}$ in space-like region}

$\begin{gathered}\text { CC cross } \\ \text { section }\end{gathered} \frac{d^{2} \sigma_{C C}\left(e^{ \pm} p\right)}{d x d Q^{2}} \propto G_{F}^{2} \times \frac{M_{W}^{4}}{\left(Q^{2}+M_{W}^{2}\right)^{2}}$

(1) $\mathbf{G}_{\mathbf{F}}-\mathbf{M}_{\mathbf{W}}-\mathbf{P D F s}$ free

\begin{tabular}{|c|c|c|}
\hline & $\mathrm{M}_{\mathrm{W}}(\mathrm{GeV})$ & $\mathrm{G}_{\mathrm{F}}\left(\mathrm{GeV}^{-2}\right)$ \\
\hline ZEUS-POL & $\begin{array}{c}82.8 \pm 1.5(\mathrm{stat}) \\
\pm 1.3(\mathrm{sys})\end{array}$ & $\begin{array}{c}(1.127 \pm 0.013(\mathrm{stat}) \\
\pm 0.014(\mathrm{sys})) \times 10^{-5}\end{array}$ \\
\hline
\end{tabular}

(2) $\mathbf{M}_{\mathrm{w}}$-PDFs free

\begin{tabular}{|c|c|}
\hline & $M_{\mathrm{W}}(\mathrm{GeV})$ \\
\hline ZEUS(HERA-I) & $\begin{array}{c}78.9 \pm 2.0 \text { (stat) } \pm 1.8(\mathrm{sys}) \\
\pm 2.0(\mathrm{PDF})\end{array}$ \\
\hline H1(HERA-I) & $\mathbf{8 2 . 8 7 \pm 1 . 8 2 ( \text { stat) } \pm 0 . 2 5 \text { (sys) }}$ \\
\hline ZEUS-POL & $79.1 \pm 0.77$ (stat) \pm 0.99 (sys) \\
\hline
\end{tabular}

$\mathrm{G}_{\mathrm{F}} \Leftrightarrow$ normalization

$\mathrm{M}_{\mathrm{W}} \Leftrightarrow \mathrm{Q}^{2}$ dependence

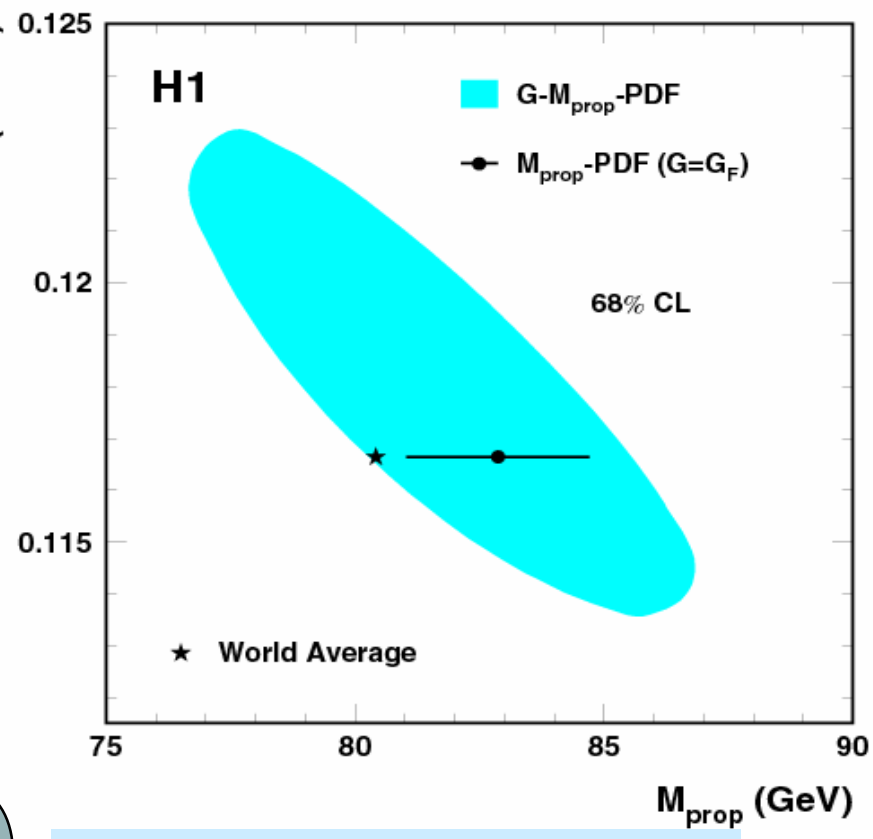

Improved by HERA-II high-statistics data

$\mathrm{G}_{\mathrm{F}}$ is consistent with one obtained from the muon lifetime measurement, and it demonstrates the universality of the CC interaction over a large range of $\mathbf{Q}^{2}$ Complementary and consistent with Tevatron/LEP time-like one 


\section{Polarized NC cross section}

$$
\frac{d^{2} \sigma^{N C}\left(e^{ \pm} p\right)}{d x d Q^{2}}=\frac{2 \pi \alpha^{2}}{x Q^{4}}\left[\left(Y_{+} \underline{F_{2}^{0}} \mp Y_{-} \underline{x F_{3}^{0}}\right)+P_{e}\left(Y_{+} \underline{F_{2}^{p}} \mp \underline{x F_{3}^{p}}\right]\right.
$$

( $F_{L}$ is ignored)

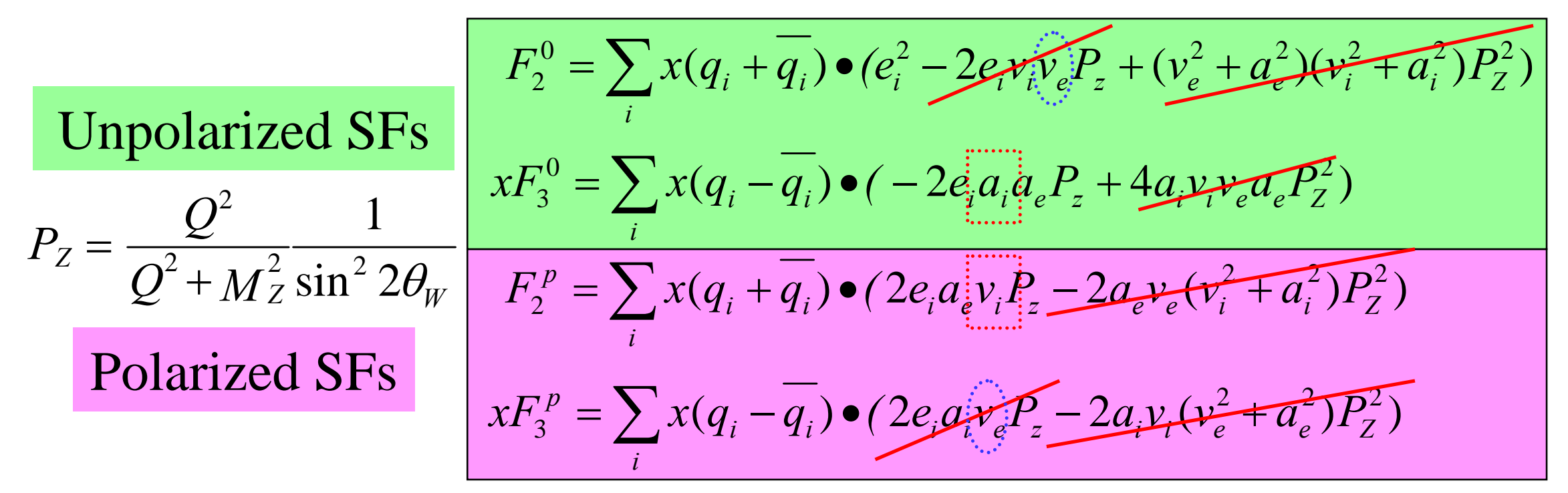

In SM,

Axial coupling $: a=T^{3}$

Vector coupling : $v=T^{3}-2 e \sin ^{2} \theta_{W}$

$$
\mathrm{v}_{\mathrm{e}} \sim \mathbf{0 . 0 4}, \mathrm{P}_{\mathrm{Z}}>>\mathrm{P}_{\mathrm{Z}}{ }^{2}
$$

$\mathrm{xF}_{3}{ }^{0}$ is sensitive to $a_{i}$

$F_{2} p$ is sensitive to $v_{i}$ 


\section{Extraction of quark couplings to $\mathrm{Z}$ only using unpolarized data}

In HERA, the light quarks dominate the cross sections, so such measurements are complementary to LEP and CDF results
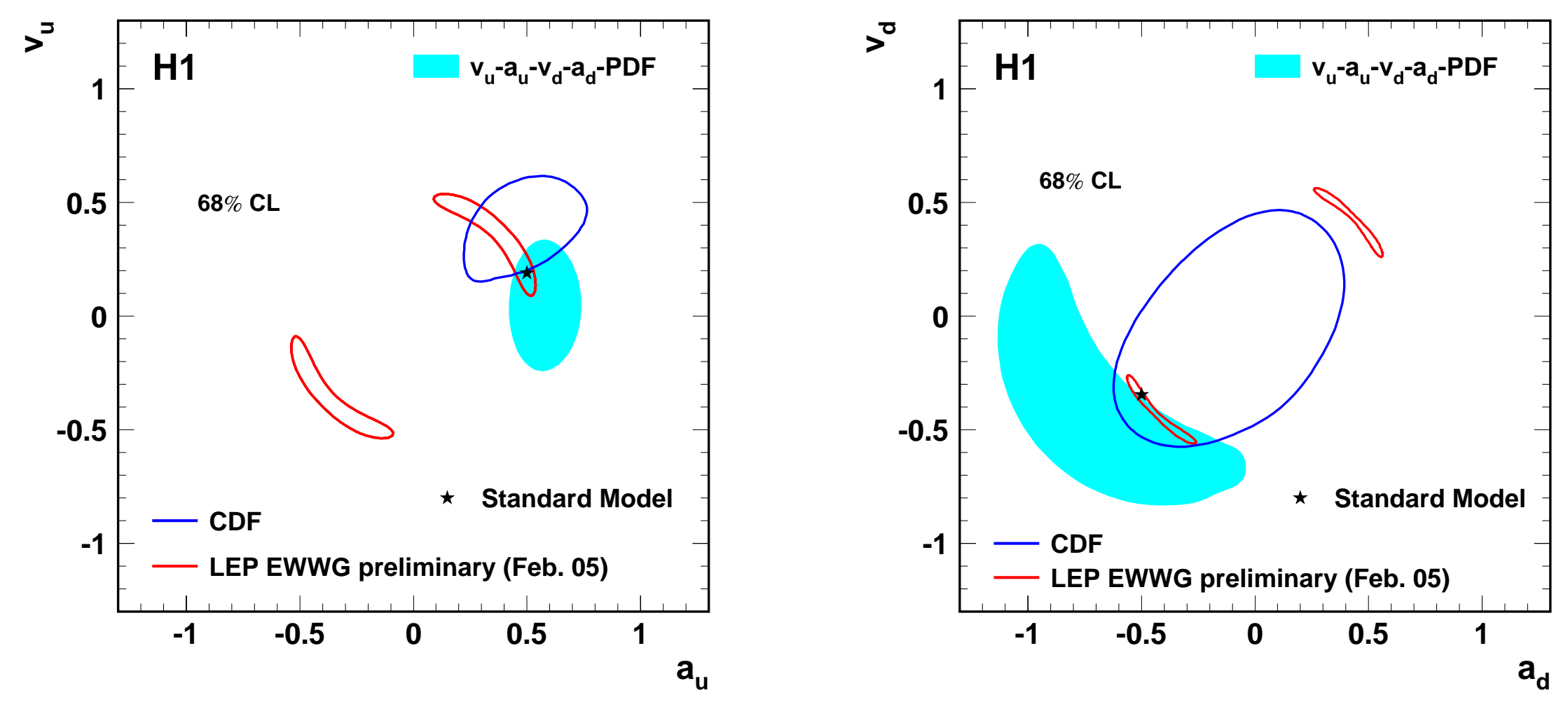

-Comparable precision to that from the Tevatron

-Remove LEP ambiguities 


\section{Extraction of quark couplings to $\mathrm{Z}$ with polarized data}

HERA-II data can improve both couplings. axial-vector : high-statistics, vector : polarized beams ZEUS

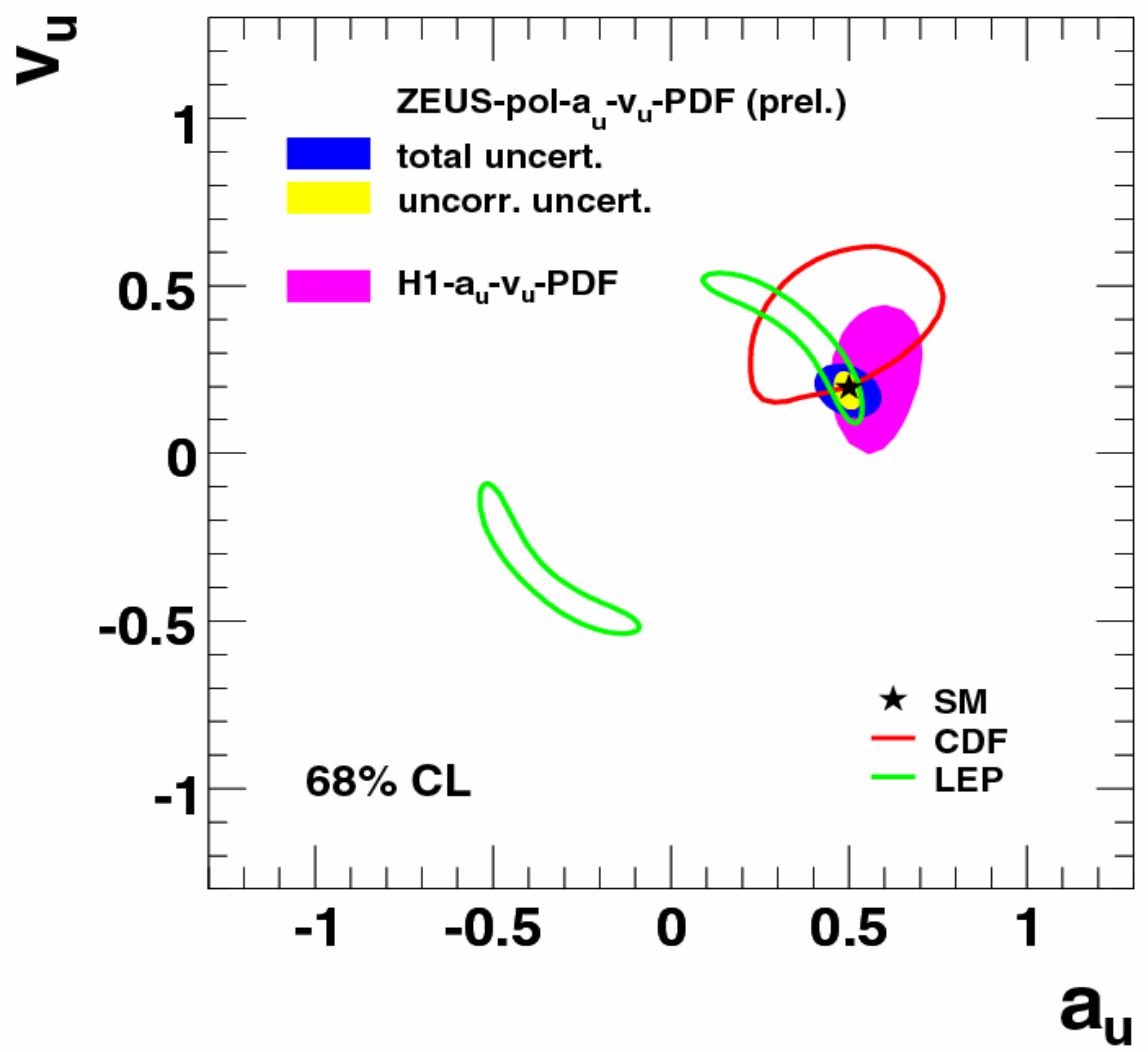

ZEUS

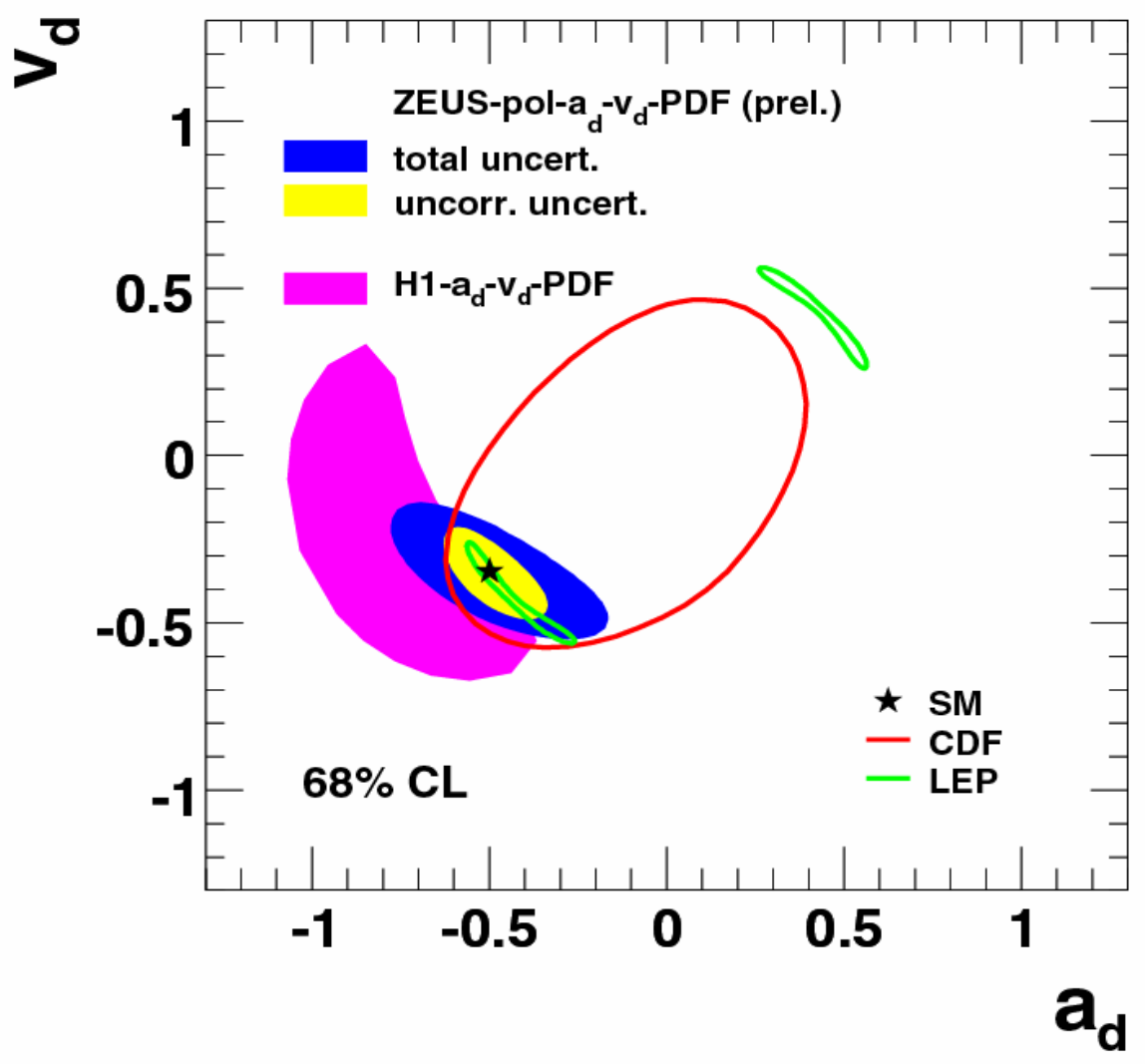

Clearly, Polarized data improves the vector couplings.

HERA-II data makes a significant impact on the quark couplings 


\section{SM formalism}

$$
\begin{aligned}
& a_{q}=T_{q}^{3} \\
& v_{q}=T_{q}^{3}-2 e_{q} \sin ^{2} \theta_{W}
\end{aligned}
$$

The values of $\mathrm{T}_{\mathrm{u}}{ }^{3}, \mathrm{~T}_{\mathrm{d}}{ }^{3}$ and $\sin ^{2} \theta_{\mathrm{W}}$ were freed with PDFs parameters.

Note: $\sin ^{2} \theta_{\mathrm{W}}$ is also present in $\mathrm{P}_{\mathrm{Z}}$, thus providing an extra constraint

\begin{tabular}{|c|c|}
\hline & ZEUS-POL- $\mathrm{T}_{\mathrm{u}}{ }^{3} \mathrm{~T}_{\mathrm{d}}{ }^{3}-\sin ^{2} \theta_{\mathrm{w}}$ \\
\hline $\mathrm{T}_{\mathrm{u}}{ }^{3}$ & $0.47 \pm 0.05 \pm 0.13$ \\
\hline $\mathrm{T}_{\mathrm{d}}{ }^{3}$ & $-0.55 \pm 0.18 \pm 0.35$ \\
\hline $\sin ^{2} \theta_{\mathrm{w}}$ & $0.231 \pm 0.024 \pm 0.070$ \\
\hline
\end{tabular}

Consistent with their SM values

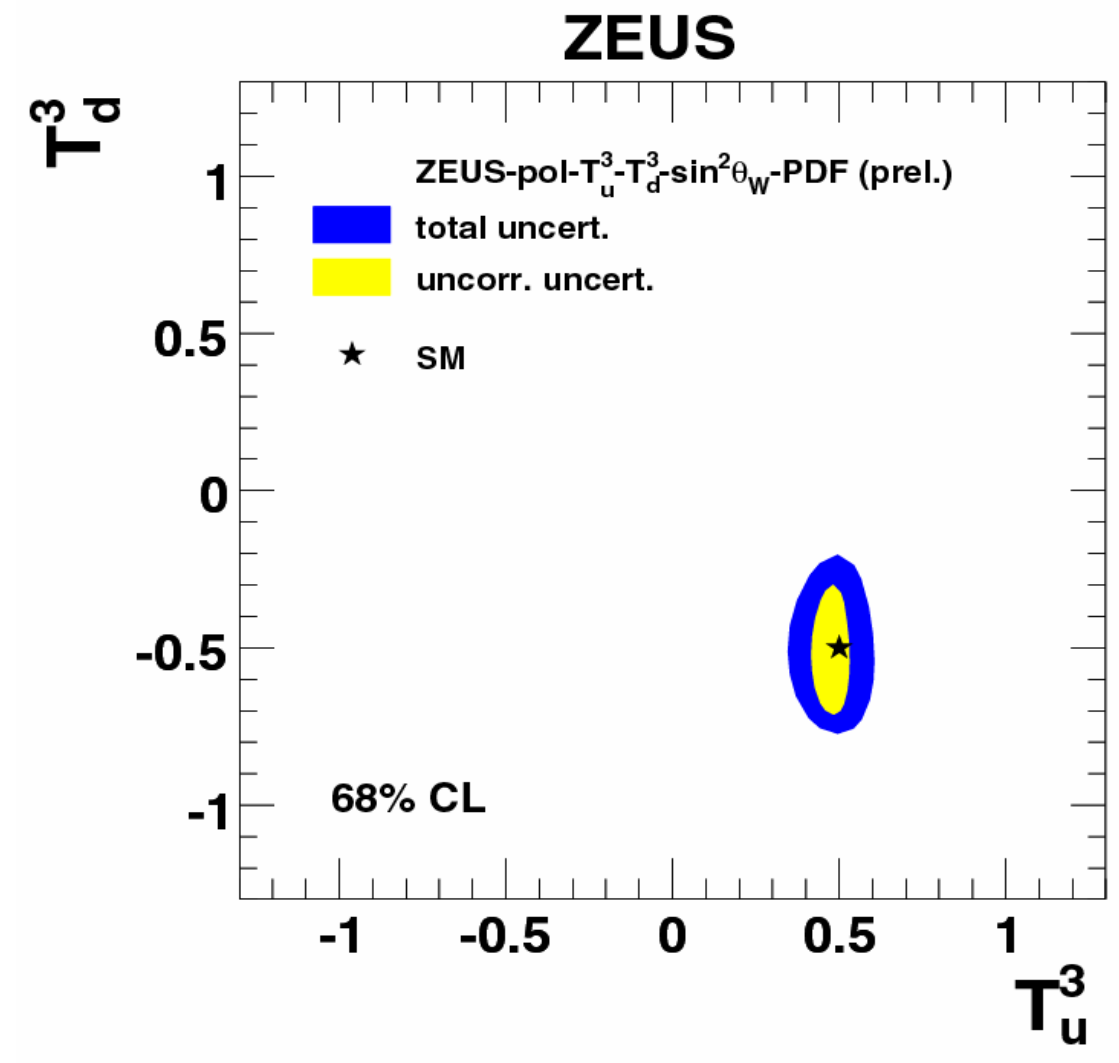




\section{Summary}

The simultaneous determination on PDFs and EW parameters can be made at HERA.

HERA-II data, large luminosity with polarized electron beams, reduced high-x PDFs uncertainties, and significantly improved the $\mathrm{EW}$ parameters.

\section{Outlook}

HERA will run until 30/June/2007 to collect polarized positron data.

Further precise measurements will come soon! 


\section{Backup slides}




\section{$a_{u}-v_{u}$ and $a_{d}-v_{d}$}

Two of the vector and axial vector couplings for $u$ and $d$ quarks are freed with PDFs parameters.
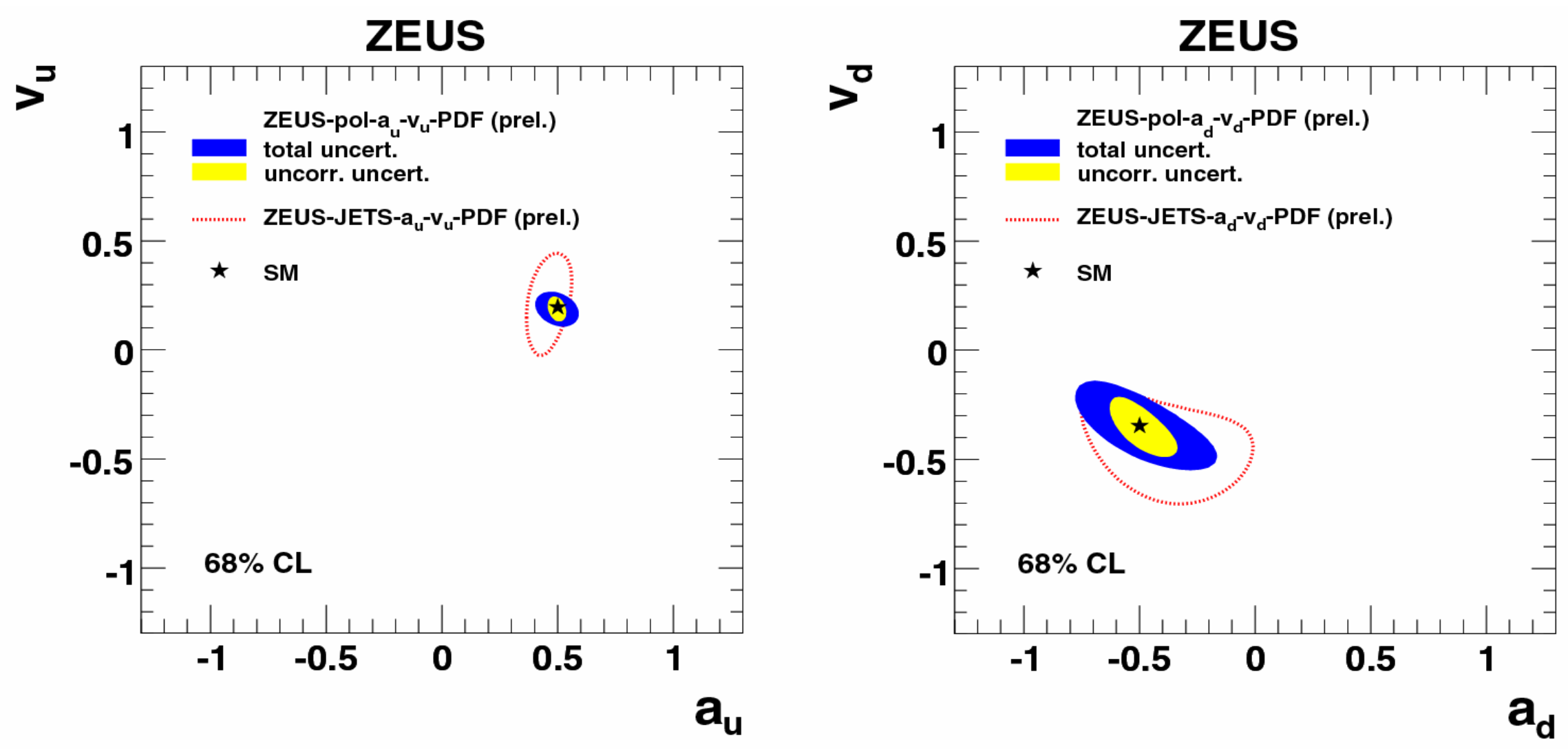

Clearly, Polarized data improves the vector couplings 


\section{$\mathrm{a}_{\mathrm{u}}-\mathrm{a}_{\mathrm{d}}$ and $\mathrm{v}_{\mathrm{u}}-\mathbf{v}_{\mathrm{d}}$}

LEP and CDF access information on $\mathrm{a}_{\mathrm{i}}{ }^{2}+\mathrm{v}_{\mathrm{i}}{ }^{2}$

But, HERA access information on $\mathrm{a}_{\mathrm{u}}$ and $\mathrm{a}_{\mathrm{d}}$ from $\mathrm{xF}_{3}{ }^{0}$, and on $\mathrm{v}_{\mathrm{u}}$ and $\mathrm{u}_{\mathrm{d}}$ from $\mathrm{F}_{2}{ }^{\mathrm{P}} \rightarrow$ stronger correlations between a flavor

ZEUS

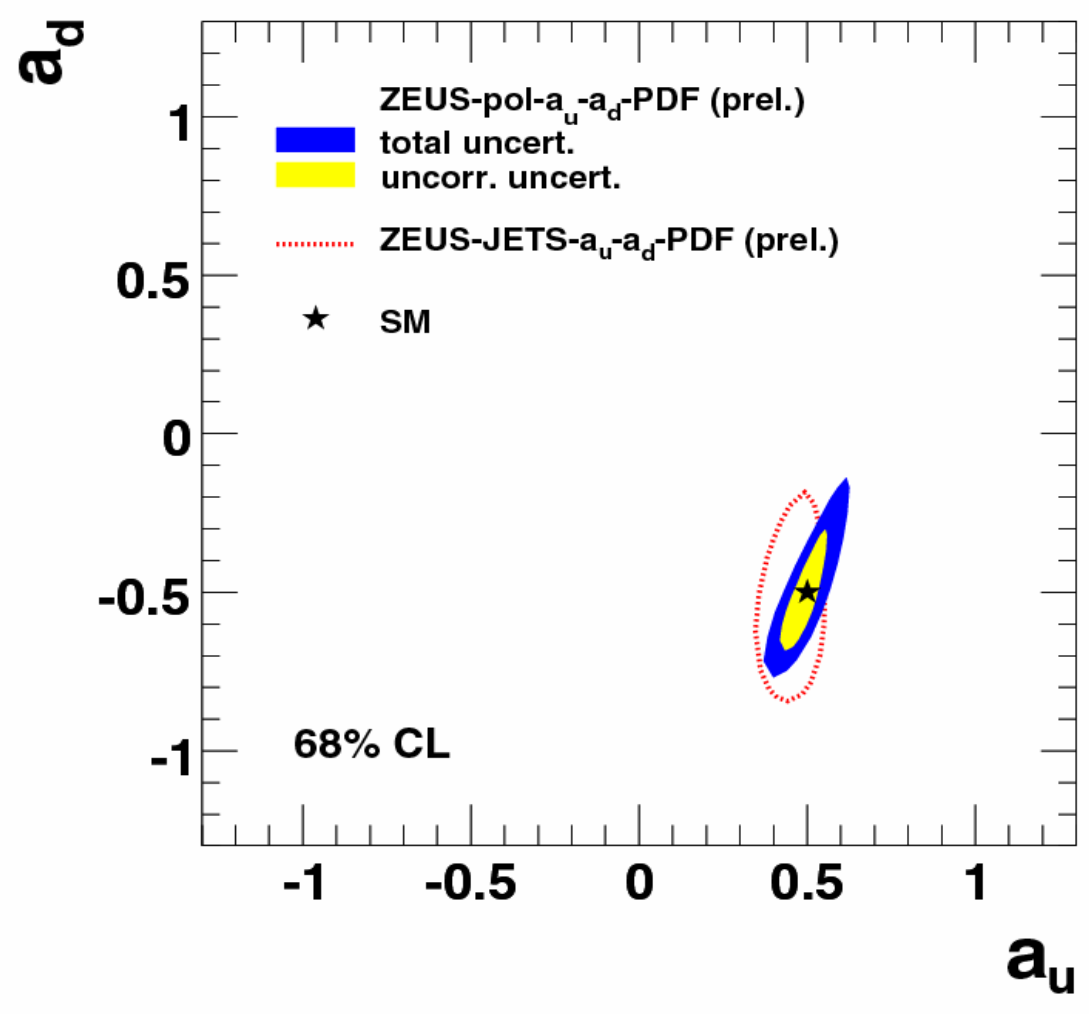

ZEUS

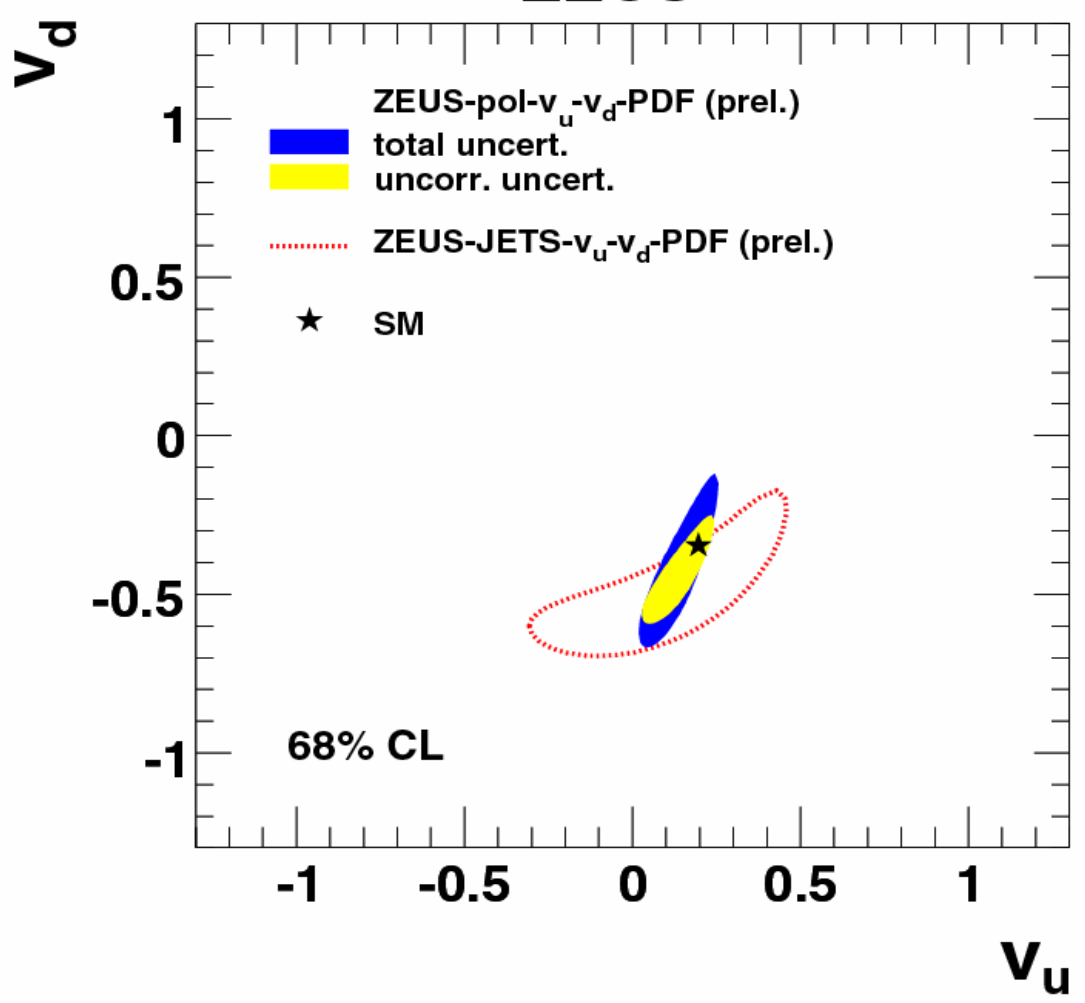

Improvement is evident 


\section{Extension of SM formalism}

\begin{tabular}{|c|c|c|c|c|}
\hline \multicolumn{4}{|c|}{$\begin{array}{l}a_{q}=T_{q, L}^{3}+T_{q, R}^{3} \\
v_{q}=T_{q, L}^{3}-T_{q, R}^{3}-2 e_{q} \sin ^{2} \theta_{W}\end{array}$} & $\theta_{W}$ \\
\hline \multirow{3}{*}{\multicolumn{2}{|c|}{ HERA-I }} & & \multirow{2}{*}{${ }_{-0.5}^{\mathrm{H} 1 \mathrm{C}}$} & \multirow{2}{*}{$\mid \begin{array}{ll} \\
\\
\end{array}$} \\
\hline & & & & \\
\hline & \multicolumn{4}{|c|}{ ZEUS-POL- $T_{u, R}{ }^{3}-T_{d, R}{ }^{3}$} \\
\hline $\mathrm{T}_{\mathrm{u}, \mathrm{R}}{ }^{3}$ & \multicolumn{4}{|c|}{$-0.04 \pm 0.06 \pm 0.13$} \\
\hline $\mathrm{T}_{\mathrm{u}, \mathrm{L}}{ }^{3}$ & \multicolumn{4}{|c|}{$-0.14 \pm 0.18 \pm 0.33$} \\
\hline & \multicolumn{4}{|c|}{ ZEUS-POL-T $\mathrm{T}_{\mathrm{u}, \mathrm{R}}{ }^{3}-\mathrm{T}_{\mathrm{d}, \mathrm{R}}{ }^{3}-\sin ^{2} \theta_{\mathrm{w}}$} \\
\hline $\mathrm{T}_{\mathrm{u}, \mathrm{R}}{ }^{3}$ & \multicolumn{4}{|c|}{$-0.07 \pm 0.07 \pm 0.07$} \\
\hline $\mathrm{T}_{\mathrm{u}, \mathrm{L}}{ }^{3}$ & \multicolumn{4}{|c|}{$-0.26 \pm 0.19 \pm 0.19$} \\
\hline $\sin ^{2} \theta_{\mathrm{w}}$ & \multicolumn{4}{|c|}{$-0.238 \pm 0.011 \pm 0.023$} \\
\hline
\end{tabular}

The extension to include righthanded isospin.

The left-handed couplings $\mathrm{T}_{\mathrm{q}, \mathrm{L}}{ }^{3}$ were fixed to SM values but the right handed couplings were freed.

$\sin ^{2} \theta_{\mathrm{w}}$ was also freed in a further fit

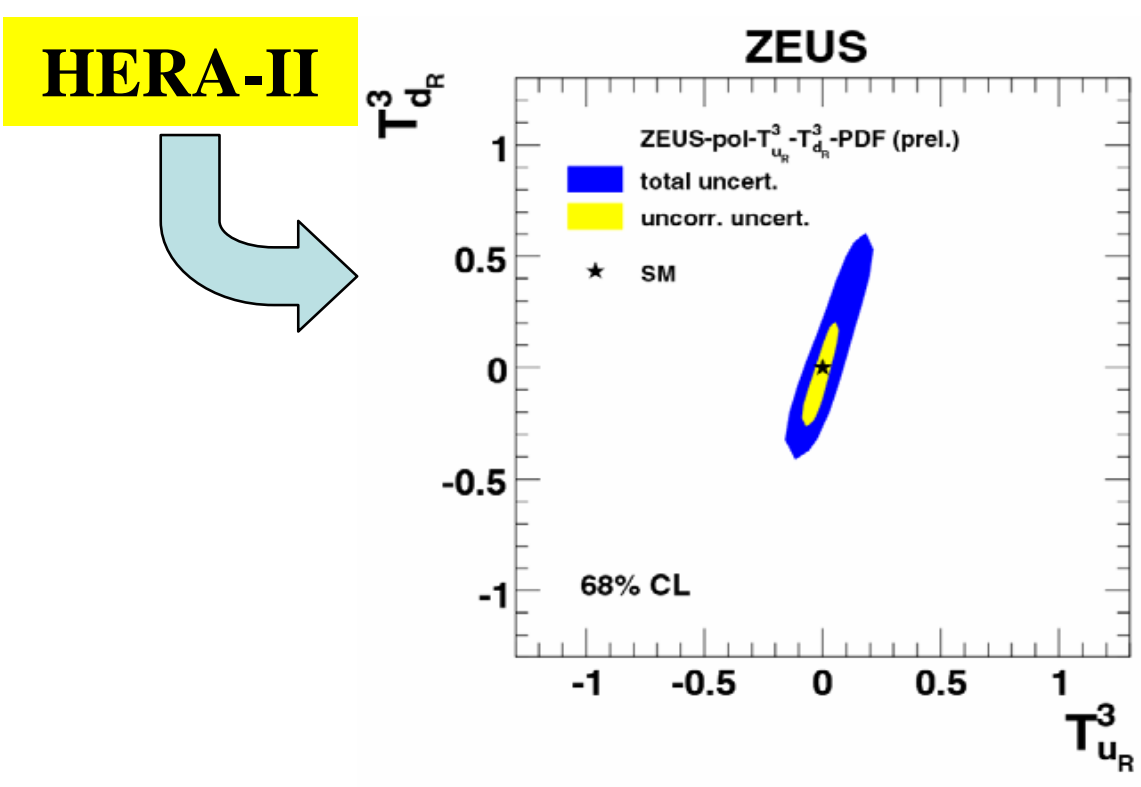

Consistent with their SM values 